\title{
BMJ Open Quality Electronic medical record alert improves HCV testing for baby boomers in primary care setting: adults born during 1945-1965
}

$\overline{\text { Eyad Al-hihi, }{ }^{1} \text { Caylin Shankweiler, }{ }^{1} \text { David Stricklen, }{ }^{2} \text { Cheryl Gibson, }{ }^{3} \text { Winston Dunn }{ }^{4}}$

To cite: Al-hihi E,

Shankweiler C, Stricklen D, et al. Electronic medical record alert improves HCV testing for baby boomers in primary care setting: adults born during 1945-1965.BMJ Open Quality 2017;6:e000084. doi:10.1136/ bmjoq-2017-000084

Received 14 April 2017 Revised 25 August 2017 Accepted 24 September 2017

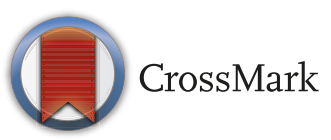

${ }^{1}$ Department of General and Geriatric Medicine, The University of Kansas Health System, Kansas, USA

${ }^{2}$ Department of Organizational Improvement, The University of Kansas Health System, Kansas, USA

${ }^{3}$ Department of Internal Medicine, The University of Kansas Medical Center, Kansas, USA

${ }^{4}$ Department of Gastroenterology and Hepatology, The University of Kansas Health System, Kansas, USA

Correspondence to Caylin Shankweiler, Department of General Internal Medicine, The University of Kansas Health System, Kansas 66160, USA; cshankweiler@kumc.edu

\section{ABSTRACT}

Method This project aims to implement the HCV birth cohort screening guidelines over a 9-month period in the primary care setting at the University of Kansas Health System General Internal Medicine Division.The project team measured the number of patients in the baby boomer population who received a one-time screen for HCV. An electronic medical record (EMR) intervention was implemented to identify baby boomers who did not have an HCV screening or diagnosis. Additionally, education was provided to all primary care providers in the clinic to increase awareness of the HCV birth cohort screening. Results The quality improvement methods increased the percentage of baby boomers who obtained a one-time screening test for HCV from a baseline of $30 \%$ to a $55 \%$ screening rate during the nine-month project period. Conclusion Identifying the HCV screening needs and creating a visual reminder in the EMR can be used to facilitate sustainable awareness and improvement of screening rates. The project team recognizes that continued work is required to close the HCV screening care gaps in the primary care setting.

\section{PROBLEM}

Approximately 3.5 million people $(1.3 \%)$ in the USA are infected with hepatitis $\mathrm{C}$ virus (HCV). ${ }^{1}$ The current generation of directacting antiviral agents (DAA) is highly effective. ${ }^{1}$ Screening, referral and treatment is the rate-limiting factor in HCV eradication. To improve screening rates, the Centers for Disease Control and Prevention (CDC) has expanded risk-based screening to include a one-time 1945-1965 birth cohort 'Baby Boomer' screening. ${ }^{2}$

The University of Kansas Health System (UKHS) General Internal Medicine Division is located in the greater Kansas City area. The clinic serves a diverse population of patients with approximately 121 patients arriving to the general medicine clinic each day, and of those patients about 58 are baby boomers.

To understand the extent of the HCV screening issue, the project team examined baseline data and identified that only $30 \%$ of the eligible population had been screened for
HCV. Subsequently, we conducted a power analysis estimating minimum detectable effect size (MDES), the smallest change in screening rate for which this study can achieve statistical significance with $80 \%$ power at 0.05 alpha level. ${ }^{3}$ Based on our historical data, we assumed 5500 eligible patients and used the baseline screening rate of $30 \%$ for the analysis. The estimated MDES was small, indicating that this study will be adequately powered $(>90 \%)$ if an observed increase in screening rate is at least $2 \%$ (ie, $>32 \%$ screening rate). Thus, the project team aimed to increase screening rates from $30 \%$ to $50 \%$, a clinically meaningful and statistically significant increase, in the eligible clinic population over a 9-month period based on reports by other investigators who implemented non-intrusive strategies to increase $\mathrm{HCV}$ screening rates in primary care practices. ${ }^{4}$

The development of visual reminders in the electronic medical record (EMR) (Epic Systems; Epic O2, Verona, WI), flagging all patients in the 1945-1965 birth cohort for one-time HCV screening was implemented. The project team measured subsequent $\mathrm{HCV}$ screening rates, monitored the implementation of the EMR alerts and notified primary care providers of their screening rates and eligible population reports on a quarterly basis.

\section{BACKGROUND}

Hepatitis $\mathrm{C}$ is a common blood-borne infection in the USA and it is reported that approximately $65 \%-75 \%$ of patients with acute HCV develop a chronic infection. ${ }^{5}$ With recent advancements, the current generation of DAAs provides a virologic cure for most patients with HCV. ${ }^{1}$ However, HCV remains underdiagnosed and undertreated, leaving approximately half of the infected population undiagnosed and only $9 \%$ of those diagnosed successfully treated. ${ }^{2}$ 
Since 1998, the CDC has recommended risk-based HCV screening, and it has been shown that in the most ideal situation, the risk-based screening will miss two-thirds of cases. ${ }^{6}$ Risk-based screening has been highly variable with inconsistent screening rates because of both patient-limiting factors such as poor social determinants of health and provider-limiting factors such as lack of knowledge regarding these risk-based guidelines. ${ }^{7}$ In 2012, the CDC expanded screening guidelines to cover the birth cohort of baby boomers to complement the current risk-based screening recommendations. ${ }^{2}$ Baby boomers account for one-fourth of the US population but they bear the burden of three-fourths of all HCV cases in the USA. ${ }^{2}$ As HCV is a chronic progressive disease and cirrhotic complications do not usually present until the third and fourth decades into the disease, baby boomers are at the highest risk for cirrhotic complications and hepatocellular carcinoma. ${ }^{7}$

The CDC has developed a variety of communication materials to educate both provider and patients, leading to inspiring examples of state successes and approaches for leveraging collaborations in care and surveillance for implementation of CDC's testing recommendations. ${ }^{68-} 10$ For example, New York State Department of Health currently requires healthcare providers to offer a one-time HCV screening test to all persons born between 1945 and 1965, mirroring the CDC's recommendations. ${ }^{10}$ Health departments located in Arkansas ${ }^{8}$ and Wisconsin ${ }^{9}$ provide funding for one-time HCV screening and education to raise awareness of HCV prevalence in the baby boomer population.

Despite these efforts, approximately $50 \%$ of persons infected with HCV are unaware of their infections and significant barriers that impede the implementation of birth cohort testing in primary care settings have been reported. ${ }^{11} 12$ The Agency for Healthcare Research and Quality recognises that engaging primary care practices in quality improvement activities is essential to improving the health of the population, enhancing patient outcomes and improving the provider experience. ${ }^{13}$ Continuous quality improvement activities are crucial to increasing the rates of HCV screening, making necessary adjustments to address unanticipated barriers and successfully implementing strategies to perform birth cohort testing. Accordingly, we sought to conduct a quality improvement project to enhance HCV screening rates in our clinic setting.

\section{MEASUREMENT}

Clinical data collections were obtained using the Clarity Database, a system that copies all reportable data from the EMR. These clinical data are then merged with demographic data from SQL Server Reporting Services to extract care gap opportunities for baby boomers who have not had HCV screening or diagnosis. For the purpose of this quality improvement project, all data have been deidentified. The eligible population was defined as patients of the UKHS General Internal Medicine
Division born between 1945 and 1965. Patients who have had a history of HCV identified by positive antibody, positive viral load or positive genotype were considered to have received screening. In addition, patients who had been screened previously, using anti-HCV, were also considered to have met the screening criteria. Rate of screening is calculated based on the number of patients screened and the eligible clinic population during each time period.

Overall, the quality improvement project was designed to improve screening rates significantly after the EMR intervention was implemented and education was provided to the primary care providers. The initial data for the study were gathered from the UKHS General Internal Medicine Division beginning on 1 June 2016. Data were extracted from the EMR on a quarterly basis and distributed to the providers to increase awareness of HCV screening rates and guidelines.

\section{DESIGN}

It is evident that the HCV infection is affecting the practice of the primary care physicians and an intervention for screening guidelines must be developed. A team of key stakeholders including a primary care physician champion, hepatology physician champion, information technology (IT) representatives and other healthcare team members developed a workflow. This workflow included visual reminders in the EMR as best practice alerts, health maintenance overdue items, along with vaccinations, and reminders for breast, colorectal and cervical cancer screenings that were developed by IT and approved by the Primary Care Steering Committee. The best practice alerts and health maintenance alerts flagged all patients in the 1945-1965 birth cohort for HCV screening. Patients who had a history of HCV identified by positive antibody, positive viral load or positive genotype were considered to have received screening. In addition, patients who had been screened previously, using anti-HCV, also satisfied the measure. If a patient was identified in clinic as meeting the criteria for HCV screening, then the provider explained the reason for the screen to the patient, ordered the anti-HCV lab and instructed the patient to have the lab drawn.

The project team met monthly to discuss additional provider education that could be delivered to increase awareness in the primary care clinics regarding HCV screening and best practice recommendations. As part of continuous quality improvement efforts, the team monitored the implementation of the EMR alerts and notified providers of their screening rates and eligible population reports on a quarterly basis. New providers and clinic staff received orientation from the clinic director or nurse manager regarding the screening process and the new providers were included in the quarterly screening numbers and eligible population reports. 


\section{STRATEGY}

Our aim was to improve screening rates of HCV over a 9-month period in UKHS General Internal Medicine Division so that $50 \%$ of eligible patients are tested for the hepatitis C infection and to be congruent with the CDC's testing recommendations. This study was conducted at a multiphysician practice, representing 84 faculty physicians and residents in the Midwest that serves as a referral centre for six surrounding counties. Baseline data were gathered in June 2016, identifying 1674 patients of the 5441 eligible patients had been screened for HCV (30\% screening rate). In lieu of this baseline data, we conducted two Plan-Do-Study-Act (PDSA) test cycles to reach our aim of a clinically meaningful and statistically significant increase in the eligible clinic population.

PDSA cycle 1: The analysis of the baseline data led to the development of PDSA cycle 1 from the months of June 2016 to August 2016. Our initial intervention was to create best practice and health maintenance overdue alerts in the EMR. This intervention was approved by the Primary Care Steering Committee for implementation and went live on 1 August 2016 and continued throughout the course of the project. These visual reminders in the EMR achieved an increase in screening rates to $45 \%$ within the first 3 months. Feedback from providers indicated visual reminders were a sustainable way to screen eligible patients but additional education, patient awareness and nurse empowerment might be helpful to improving screening rates.

PDSA cycle 2: To provide education to the primary care providers, the UKHS General Internal Medicine
Division invited their colleagues in Gastroenterology and Hepatology to provide education regarding birth cohort screening for HCV. The providers received a single in-person education session from the Division of Gastroenterology and Hepatology at the start of December 2016. This education piece in conjunction with the EMR alerts achieved a $10 \%$ increase in screening rates over the next 3 months, improving the screening rate to $55 \%$ by March 2017. Feedback from providers indicated that additional patient awareness and nurse empowerment might encourage further improvements to screening rates. Currently, our improvement team continues to monitor HCV screening rates, and plans to further act on the feedback provided during the second PDSA cycle.

\section{RESULTS}

Our outcome measure goal was to increase HCV screening rates for the patient population of the UKHS General Internal Medicine Division clinic using birth cohort testing recommended by the CDC. We successfully implemented this strategy and achieved our goal with screening rates increasing from $30 \%$ to $55 \%$ during the two planned cycles of the quality improvement project (see figure 1).

Each subsequent intervention that was implemented had a significant effect on screening rates. As displayed, the intervention during PDSA cycle 1 that was implemented in August 2016 and evaluated in December 2016 was effective $(45 \%)$ over its 3-month trial in comparison to baseline data (30\%) measured in June 2016. Similarly, evidence from the graph also shows the intervention

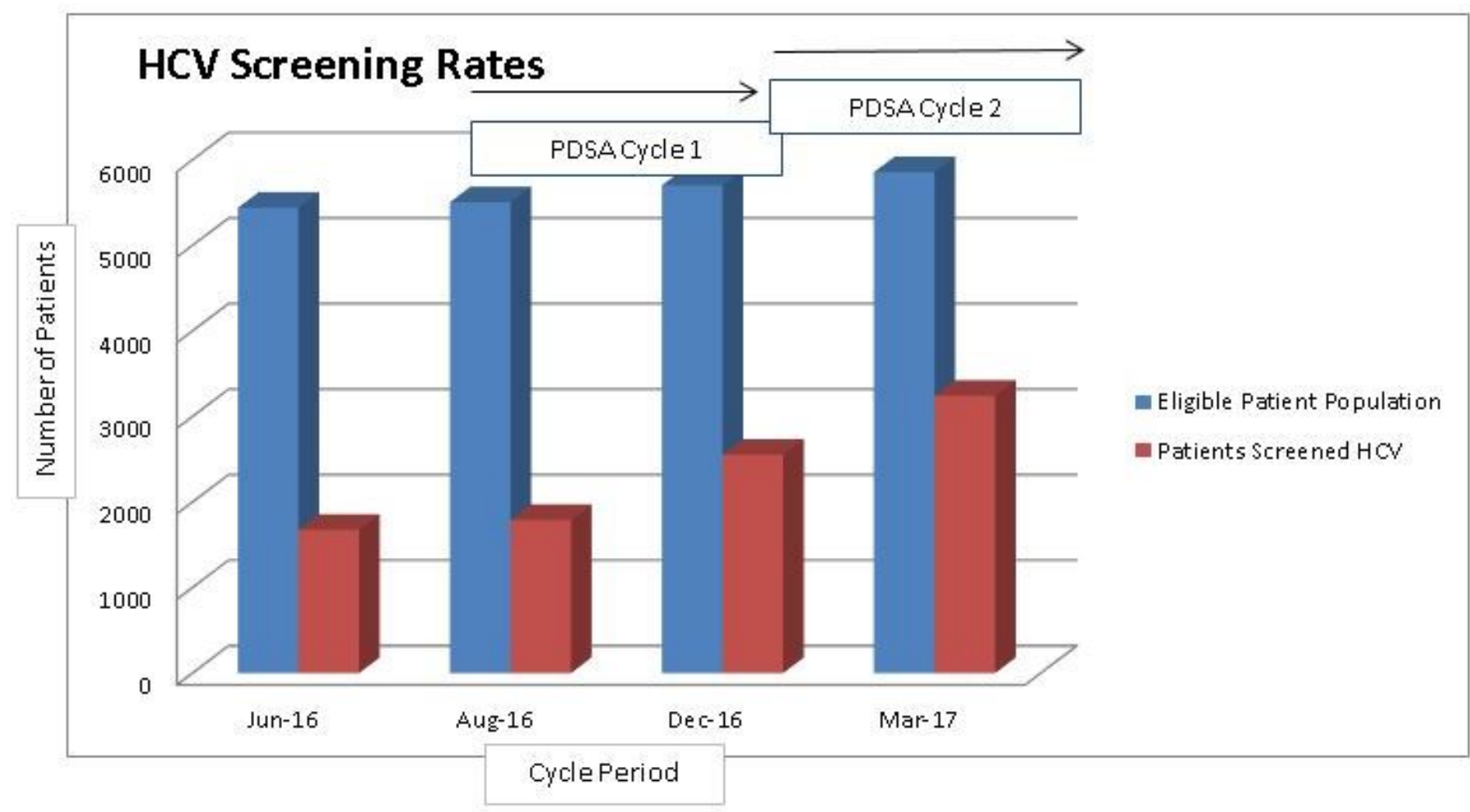

Figure 1 HCV screening rates. HCV, hepatitis C virus; PDSA, Plan-Do-Study-Act. 


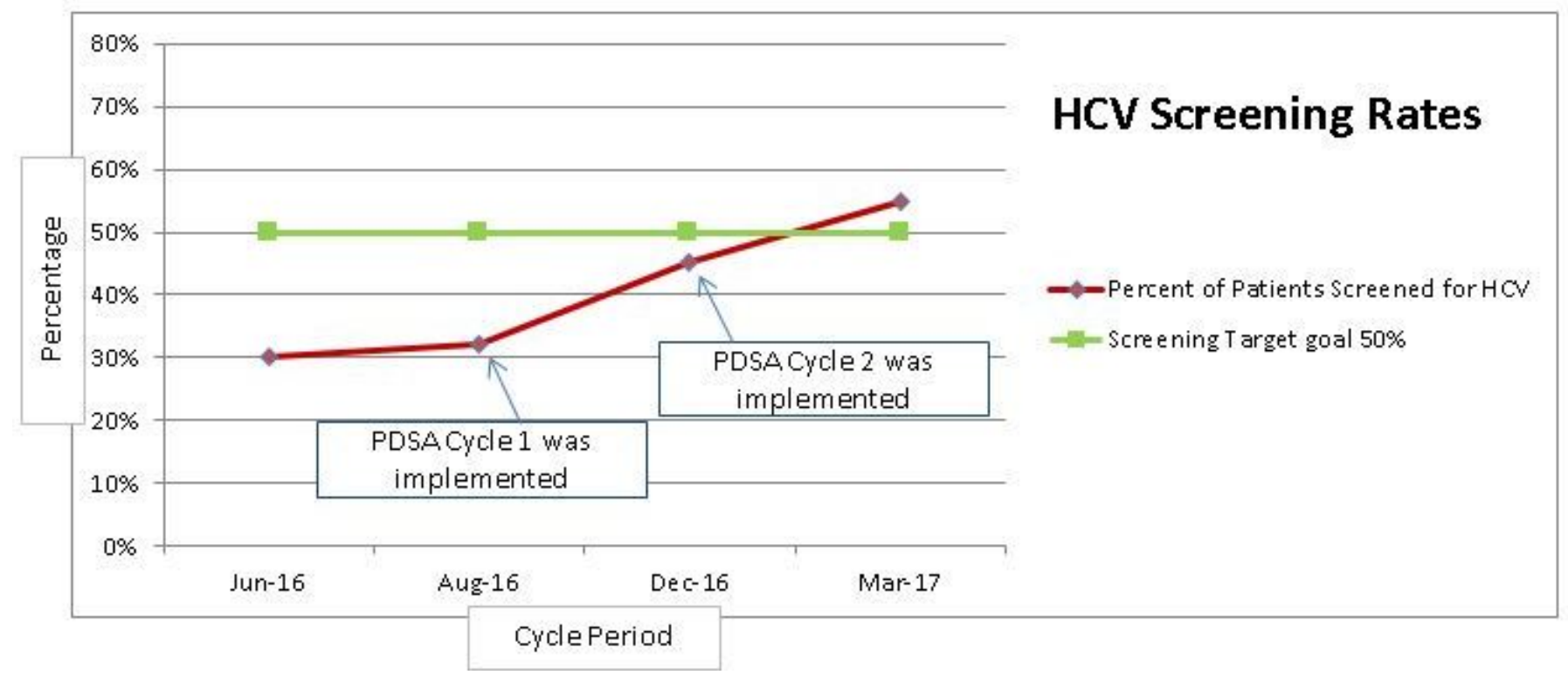

Figure 2 HCV screening target. HCV, hepatitis C virus; PDSA, Plan-Do-Study-Act.

from PDSA cycle 2 implemented in December 2016 in conjunction with the EMR alerts was also effective (55\%) by March 2017, ending the 9-month trial (see figure 2).

\section{LESSONS AND LIMITATIONS}

Our findings indicate that the implementation of the 1945-1965 birth cohort testing in accordance with the CDC, using EMR reminders and continuous education, was successful in increasing screening rates. The use of the EMR was most effective because it involved visual reminders to cue HCV screening needs of patients who fit the baby boomer testing criteria. By adding EMR reminders to the providers' workflow, we were able to identify care gap opportunities easily. Additionally, because health maintenance and best practice alerts were already a part of this workflow, the probability of sustaining this screening effort over time is much more plausible.

Additional lessons included making sure to include open discussions about what changes might enhance future screening interventions and incorporating the feedback received from care providers. At the end of the second PDSA cycle, providers suggested increasing the involvement of nurses and engaging patients in the quality improvement activities. From this feedback, our team is in the process of developing a third PDSA cycle. The plan currently under development is to increase nurses' engagement by utilising a nurse protocol for ordering the HCV screen for the eligible patient population. Further, educational flyers on the importance of HCV screening will be presented in the patient waiting rooms in hopes of generating meaningful dialogue between patients and their primary care provider. After implementation of these interventions, beginning on 1 April 2017, we plan to reassess screening rates in June 2017.

Our study has some limitations. Because this project was conducted at a single centre, our findings may not be generalisable, especially among hospital systems that have not adopted EMR for clinical patient care. Future efforts should expand the HCV screening project to include multiple providers across different settings to determine if our findings can be replicated.

Additionally, while the HCV screening rates continue to improve, it would have been beneficial to evaluate referral and treatment outcomes of those who were diagnosed with hepatitis $\mathrm{C}$ during the screening process. To help ensure that identified cases receive referral to subsequent care, our project team plans to identify potential referral and treatment gaps as our next steps in this care opportunity.

\section{CONCLUSION}

In summary, the 1945-1965 birth cohort testing can result in the identification of those who test positive for hepatitis $\mathrm{C}$ and can be easily implemented in the primary care setting. Although primary care providers can get overwhelmed with multiple patient screening requirements, the implementation of this one-time HCV screening among baby boomers is a simple, effective way to address this barrier.

The project team was able to meet the aim of improving $\mathrm{HCV}$ screening rates above $50 \%$ in the General Internal Medicine Division clinic setting. The intervention, which consisted of EMR alerts and education, enabled the screening rates to increase substantially, although continued work is required to maintain optimal levels of screening to close the clinic's HCV screening care gaps.

Due to the positive outcome gained by this project and how well received it was by the care providers, we plan to share these strategies throughout primary care settings so that patients who likely would not be diagnosed and treated until they present with complications can be identified. The project team has begun supporting additional interventions within the General Internal Medicine Division to track continuous improvement of HCV screening 
rates. In addition, linkage to care work is under way with the development of tracking referrals and treatment outcomes for patients with a positive HCV screen in the ambulatory care setting.

Contributors CS takes primary responsibility for communication with the journal during the manuscript submission, peer review and publication process.

Competing interests None declared.

Ethics approval The quality improvement protocol was approved by the Institutional Review Board of the University of Kansas Medical Center.

Provenance and peer review Not commissioned; externally peer reviewed.

Open Access This is an Open Access article distributed in accordance with the Creative Commons Attribution Non Commercial (CC BY-NC 4.0) license, which permits others to distribute, remix, adapt, build upon this work non-commercially, and license their derivative works on different terms, provided the original work is properly cited and the use is non-commercial. See: http://creativecommons.org/ licenses/by-nc/4.0/

(C) Published by the BMJ Publishing Group Limited. For permission to use (where not already granted under a licence) please go to http://www.bmj.com/company/ products-services/rights-and-licensing/

\section{REFERENCES}

1. Edlin BR, Eckhardt BJ, Shu MA, et al. Toward a more accurate estimate of the prevalence of hepatitis $C$ in the United States. Hepatology 2015;62:1353-63.

2. Yehia BR, Schranz AJ, Umscheid CA, et al. The treatment cascade for chronic hepatitis $C$ virus infection in the United States: a systematic review and meta-analysis. PLoS One 2014;9:e101554.
3. Bloom HS. Randomizing groups to evaluate place-based programs. In.Bloom HS, ed. Learning more from social experiments: evolving analytic approaches. New York: Russell Sage Foundation, 2005:115-72.

4. Sidlow R, Msaouel P. Improving hepatitis $C$ virus screening rates in primary care: a 298 targeted intervention using the electronic health record. J Healthcare Quality 2015;37:319-23.

5. Ditah I, Ditah F, Devaki P, et al. The changing epidemiology of hepatitis $C$ virus infection in the United States: national health and nutrition examination survey 2001 through 2010. $J$ Hepatol 2014;60:691-8.

6. CDC's recommendations for One-time hepatitis $C$ virus testing for persons born during 1945-1965:public health's role and key elements for effective implementation. Association of state and territorial health officials, 2013:1-12.

7. Smith BD, Morgan RL, Beckett GA, et al. Recommendations for the identification of chronic hepatitis $C$ virus infection among persons born during 1945-1965. MMWR Recomm Rep 2012;61:1-18.

8. Arkansas promotes hepatitis $C$ testing among baby boomers. Association of 315 state and territorial health officials, 2015:1-2.

9. Wisconsin Promotes Hepatitis C Testing Among Baby Boomers. Association of state and territorial health officials, 2015:1-2.

10. Flanigan $\mathrm{C}$. Implementing the nation's first state hepatitis $C$ testing law, 2015. https://blog.aids.gov/2015/05/implementing-the-nationsfirst-state-hepatitis-c-testing-law.html

11. Younossi ZM, Stepanova M, Afendy M, et al. Knowledge about infection is the only predictor of treatment in patients with chronic hepatitis C. J Viral Hepat 2013;20:550-5.

12. Kruger DL, Rein DB, Kil N, et al. Implementation of birth-cohort testing for hepatitis $C$ virus: lessons learned from three primary care sites, 2016.

13. Quality Improvement in Primary Care. Content last reviewed. Rockville, MD: Agency for Healthcare Research and Quality, 2014. http://www.ahrq.gov/research/findings/factsheets/quality/qipc/index. html 